\title{
It depends what you do in the laboratory
}

- a response to "Cognitive Ethology"

\author{
Roger Watt \& Sandra Quinn \\ Department of Psychology, University of Stirling
}

Let us start by stating a strong opinion: psychology can only claim honesty when it makes the effort to relate its own theories and other observations about human behaviour and experience to actual human behaviour and experience in the real world.

In this respect, we agree with some of the assumptions the authors make in this article and we share with the authors a sense of frustration that so much effort in cognitive psychology can sometimes yield very little insight beyond the relative sterility shown by some laboratory phenomena.

Late in the year 1999 one of us (RJW) was asked to provide expert witness evidence to a Public Inquiry that followed a fatal train crash in London (Cullen, 2001). The fundamental question was whether there was any good perceptual explanation for a driver proceeding through a red signal when on so many other occasions he and other drivers had not got it wrong. To cut short a long story, it was found that some 5 or 6 seconds away from the signal, it would have appeared to be yellow not red for a brief moment because of a combination of optical and visual factors. However, no-one was able to explain why in the remaining time beyond that brief moment, when the signal should have reverted to appearing red, the driver didn't notice that the signal was in fact red. Although the driver would have had no reason to look at the signal again, five seconds is a long time.

Another expert witness had suggested that inhibition of return might have caused the train driver to miss the signal. On approach to the signal, but before it come into view, it was suggested that the driver may have looked in the direction of the signal. It was not there, so he didn't learn anything about the signal. However, inhibition of return would have meant that he was unlikely or unable to look in that direction again and so never saw the signal. This doesn't sound plausible, and were inhibition of return such a powerful effect no-one would travel by rail. Indeed it turns out that we must take some care with the phenomenon - the original data show a slight slowing of an eye-movement to a place that has just been inspected. The effect is small (a small fraction of a second longer latency) and occurs over a fairly brief time period of a second or so.

On the face of it, inhibition of return seems like a good instance of the type of laboratory finding that we are warned against in the Cognitive Ethology approach. On simple common-sense and introspective grounds, it seems wholly unlikely to exist or to matter. But it does exist and we are probably better off knowing about it. There's no reason to suppose that inhibition of return would not occur in the real world, just lots of reasons for thinking it would be hard to measure out there.

Now we state a second opinion: humans are so profoundly expert in the real world that studying them there can be uninformative and perhaps even misleading. A simple broad example will help. Our visual system rarely lets us down in the real world - nearly all the time it gets it right. We don't often discover lacunae in our perception of the environment - moments or places where what we see and what happens do not match. 
The problem is essentially a pragmatic one. You learn most about a computation when it comes out wrong. If you are asked what $362 \times 2$ comes to, and you say 724 then almost nothing is learnt about how you did it, except that you have used one of the large number of competent methods. If you say 624, then we know you have used a mechanism that involved carrying digits to the base 10 forward (and that you forgot to). If you say 700ish, then we know you've used a slide rule or some other device that deals in approximations. And so on.

This problem is a variant on the beautiful notion of Braitenberg (1989), which he encapsulated in the "law of uphill analysis and downhill invention". A quote suffices:

"It is pleasurable and easy to create little machines that do certain tricks. It is also quite easy to observe the full repertoire of behaviour of these machines - even if it goes beyond what we had planned, as it often does. But it is much more difficult to start from the outside and to try and guess internal structure just from the observation of behaviour."

If it is accepted that successful performance is not very useful in analysing the structure of a complex system, then it follows that one avoids places where the system displays consummate skill and instead looks for a situation where the system delivers wrong answers. For me and my area of expertise, visual perception, the laboratory wins every time. The simple reason that the laboratory works in studying vision is that the visual system makes massive assumptions about the probable or statistical nature of the world. These assumptions work in the real world. However, a carefully constructed laboratory stimulus can defeat these assumptions and reveal the mechanisms.

Figure 1 shows a stimulus that we are presently interested in. Whilst it is an unremarkable laboratory stimulus in vision, it is certainly not one that the environment has in any quantity. In that sense, it could perhaps be written off as a laboratory curiosity. However, cover up all the even numbered lines (the second, fourth etc) and you will discover that hidden in the figure is perfectly regular array of evenly spaced lines. Even when you know that the regularity is there you cannot see it. From this simple observation, we can conclude these points about the machinery of spatial vision: you cannot readily judge distances between non-adjacent features

- in addition, you cannot readily combine pairs of judgements of distances

Figure 1 about here

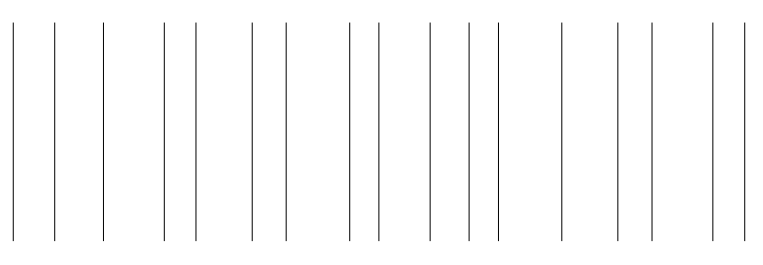

A good laboratory study is one that forces cognition to get it wrong. Errors of commission - cognition doing things it shouldn't - and errors of omission - cognition not doing things it should - are equally useful. The example in Figure 1 is an error of omission - despite there being every incentive to see the regularity you simply haven't got the machinery needed to see it: cognition has omitted this operation. 
But the authors are right in this next respect. It is not explanatory to describe the phenomenon of Figure 1 in laboratory terms such as "failure to detect second-order periodicities" (which just describe the stimulus), or in terms of a visual mechanism such as "adjacency detector" (which just describe the nearest the participant can get to doing the task). They talk (p17 of ms) clearly of the wish for "a satisfying answer as to why cognitive performance is like it is". I agree that wish. The explanation for the phenomenon of Figure 1 is

- hardly ever does the real world offer us a row of identical stimuli that are actually two interleaved independent patterns

- if ever it does, such a pattern is most unlikely to have any meaning

- consequently vision has no use for a mechanism that might use valuable resource to consider such a possibility.

This is what Marr (1982) would have called a Computational Theory, and what the authors call a "personal" level explanation. I am fully with them on this, never having recovered from the effects of reading Marr at a relatively impressionable age.

Vision may be a special case, less needing of Cognitive Ethology. One area of research where studying perception in the real world may seem to be the only sensible approach is in the perception of music. In the real world, listening to music is often a very rich personal experience and even varies from occasion to occasion. At a concert we may experience an overwhelming emotional reaction to the music, whilst on other occasions we have no response other than a basic appreciation for the same piece of music. The simple issue is whether a listener hears music in the same way in a laboratory as they do a concert or using their MP3 player. On balance, it is very likely that they don't. If they don't, then laboratory studies of music perception are not what they claim to be.

This is a strong criticism and maybe unreasonable. Studies of music perception go to extraordinary lengths to ensure that the stimuli they use are musical - sequences of notes that are broadly in line with musical practice. Studies of music perception tend to use exclusively or predominantly musical participants - formally trained or student musicians. However, they do not use listening conditions that are musical. Actually the situation is more like a facet of the real world than will be apparent. Music students will be only too familiar with being asked to listen to some musical stimulus in a classroom and make some musical judgement - that is how their ear is trained to hear musically. Presumably, they treat the perceptual laboratory as a similar experience, possibly with unwanted consequences.

Does this line of thinking rule out the use of laboratory studies to inform our understanding of music perception? We think not, but once again success in the laboratory depends on what you do there. You can't directly study the perception of hope in Mendelssohn's Fingal's Cave in the laboratory because the prospects of eliciting that percept in the laboratory are hopeless. On the other hand, if it depends (when it does happen in the concert hall) on our ability to perceive and interpret relationships between sound and time, then these at least can be studied in the laboratory. We would go further and say that they must be studied in the laboratory because it is only there that you can break the expertise of the experienced musical listener in all of us and the richness of time information, by generating deliberately impoverished stimuli.

To conclude, we propose a simple signal detection analysis of Cognitive Ethology. Let's treat valid findings as hits, invalid findings as false alarms and everything else as misses. Now for each hit, let us suppose that it can be characterized by (i) whether it can 
be discovered by laboratory work or not, and (ii) whether it can be discovered by realworld work checked by laboratory studies. There are then potentially 4 types of finding:

\begin{tabular}{|r|c|c|}
\hline & Real World & Laboratory \\
\hline Type 1 & Yes & Yes \\
\hline Type 2 & No & Yes \\
\hline Type 3 & Yes & No \\
\hline Type 4 & No & No \\
\hline
\end{tabular}

Type 1 and Type 4 are irrelevant - we can get the hits either way. If we stay in our labs, then we lose Type 3; if we stay away from our labs, or only use our labs to confirm observations made in the real world, then we lose Type 2. Now we must look at the false alarms. Exactly the same table applies. If we stay in our labs, then we risk Type 2 false alarms; if we stay away from our labs, we risk Type 3 false alarms. The optimum strategy depends on the relative probabilities, costs and benefits of Type 2 hits and Type 2 false alarms, compared with the relative probabilities, costs and benefits of Type 3 hits and Type 3 false alarms. The authors are saying that:

- $\quad$ the probability of Type 2 hits is lower than Type 3 hits

- $\quad$ the benefits of Type 2 hits are less than Type 3 hits

- $\quad$ the probability of Type 2 false alarms is greater than Type 3 false alarms

- $\quad$ the costs of Type 2 false alarms are greater than Type 3 false alarms

We agree that it is not difficult to find thoughtless laboratory studies that confirm their views. However, none of these propositions seem strongly self-evident, provided some care goes into what one does in the laboratory.

\section{References}

Braitenberg, 1989. Vehicles: experiments in synthetic psychology. MIT Press Boston. Cullen, 2001. The Ladbroke Grove Rail Inquiry Part 1 Report. Crown Copyright. HSE Books, London

Marr, 1982. Vision. Freeman, San Francisco 